\title{
Archaeonautica
}

Archaeonautica

L'archéologie maritime et navale de la préhistoire à

l'époque contemporaine

$20 \mid 2018$

De re navali : Pérégrinations nautiques entre

Méditerranée et océan Indien

\section{Quelques pièces pour la manœuvre des voiles découvertes sur l'épave de la Madrague de Giens (Hyères, Var, 70-45 av. J.-C.)}

Rigging elements found on the Madrague de Giens shipwreck (Hyères, Var, France, $1^{\text {st }}$ century $B C$ )

\section{Marie-Brigitte Carre}

\section{OpenEdition} Journals

Édition électronique

URL : http://journals.openedition.org/archaeonautica/384

DOI : 10.4000/archaeonautica.384

ISSN : 2117-6973

Éditeur

CNRS Éditions

Édition imprimée

Date de publication : 6 décembre 2018

Pagination : $57-65$

ISBN : 978-2-271-12263-6

ISSN : 0154-1854

Référence électronique

Marie-Brigitte Carre, "Quelques pièces pour la manœuvre des voiles découvertes sur l'épave de la Madrague de Giens (Hyères, Var, 70-45 av. J.-C.) », Archaeonautica [En ligne], 20 | 2018, mis en ligne le 30 avril 2020, consulté le 30 avril 2020. URL : http://journals.openedition.org/archaeonautica/384 ; DOI : https://doi.org/10.4000/archaeonautica.384 


\title{
QUELQUES PIÈCES POUR LA MANOEUVRE DES VOILES DÉCOUVERTES SUR L'ÉPAVE de la Madrague de Giens (HYÈrES, VAR, 70-45 AV. J.-C.)
}

\author{
Marie-Brigitte CARRE
}

\begin{abstract}
Résumé
Cet article présente quelques-unes des pièces de gréement trouvées sur l'épave de la Madrague de Giens (Hyères, Var, 70-45 av. J.-C.) durant les campagnes de fouille 1976 à 1982 : poulies, moques, manœuvres de voile, replacées dans le contexte de l'épave et dans celui plus général des embarcations antiques. On tentera d'identifier leur fonction par comparaison avec les agrès utilisés traditionnellement dans la marine à voile moderne.
\end{abstract}

Mots clés

Gréement, Poulie, Navire romain
Rigging elements found on the Madrague de Giens shipwreck (Hyères, Var, France, $1^{\text {st }}$ Century BC)

\section{Abstract}

This article presents some of the rigging elements that were found on the Madrague de Giens shipwreck (Hyères, Var, France, 70-45 B.C.) during the excavation campaigns of 1976 to 1982. Sheave blocks, deadeyes, and brail rings are placed within the context of the wreck and, more generally, of ancient ships. We attempt to identify their function through comparisons with fittings that are traditionally used in modern sailing ships.

\section{Key words}

Rigging, Sheave block, Roman ship
Lors de ma première campagne de fouille sur l'épave de la Madrague de Giens en 1978, la vision de ce navire magnifique et le sentiment d'entrer dans un groupe de recherche exceptionnel dirigé par André Tchernia et Patrice Pomey m'ont conduite à solliciter de Patrice un sujet de thèse. Il me confia alors l'étude du gréement et de l'équipement des navires antiques (Carre 1983). Ce travail est resté inédit, à l'exception de deux articles sur les pompes de cale (Carre, Jézégou 1984 ; Carre 2007). Trente-cinq ans après la soutenance de la thèse, ces quelques pages se veulent un rappel de l'importance fondamentale des études promues par Patrice et un affectueux hommage de la part de son ancienne élève qui a abandonné depuis l'architecture navale, mais lui a succédé à la direction d'Archaeonautica où elle a toujours pu compter sur son implication active, éclairée et bienveillante - jusqu'à ce volume où il n'a bien fallu compter que sur nous-mêmes.

Cette courte contribution ne constitue ici qu'une note très préliminaire sur une partie des pièces découvertes lors de la fouille. Leur étude complète sera intégrée dans la publication intégrale qui sera réalisée sous la direction de Patrice Pomey. Elle pourra bénéficier, comme toujours, des conseils de Patrice, anonyme coauteur de tous ceux qui ont eu la chance de travailler auprès de lui.

\section{LE NAVIRE ET SA CARGAISON}

L'épave de la Madrague de Giens a été fouillée entre 1972 et $1982^{1}$. Ce navire d'une quarantaine de mètres de long était chargé d'environ 6000 amphores contenant du vin de la plaine de Fondi

1. Une première synthèse des travaux jusqu'en 1975 a été publiée en 1978 (Tchernia et al.), suivie de plusieurs contributions portant sur des points spéci- dans le Latium et de quelques caisses de vaisselle de table et de cuisine de Campanie. Il a fait naufrage dans le deuxième quart du $\mathrm{I}^{\mathrm{er}} \mathrm{S}$. av. J.-C. près de la presqu'île de Giens, dans le Var, sans atteindre sa destination, probablement le golfe de Fos, où les marchandises qu'il transportait auraient été réacheminées vers la vallée du Rhône. Une partie de la cargaison a été récupérée par les urinatores ce qui, ajouté au naufrage, a encore contribué au bouleversement de ses parties hautes et du gréement mais, on le verra, a sans doute permis d'en conserver quelques éléments.

\section{LA DÉCOUVERTE DES PIÈCES SUR L'ÉPAVE}

Le gréement des navires antiques est, selon la définition de Bonnefoux et Pâris « l'ensemble des cordages, manœuvres, poulies et autres objets servant à l'établissement, à la tenue de la mâture, des vergues et des voiles, ainsi que la totalité des objets qui entrent à bord d'un bâtiment ». On comprend aisément pourquoi les vestiges de la mâture sont si rarement retrouvés car ils sont les premiers à disparaître lors du naufrage. On a la chance parfois d'en récupérer quelques-uns, lorsque « la rafale, rompant d'un coup les deux étais, nous renverse le mât et fait pleuvoir tous les agrès à fond de cale » (Homère, Od. 12, 408-411, trad. Bérard, CUF). Cependant ils ne sont que rarement en place et, sortis de leur contexte d'utilisation, ils sont parfois bien difficiles à identifier. L'utilisation de manuels de la marine à voile traditionnelle a souvent été fondamentale pour en comprendre la fonction et le rôle (Barrot de Gaillard 1939, Devillers 1971).

fiques, dont on ne citera que quelques-unes parmi les plus significatives : Pomey 1982, Tchernia 1988, Hesnard 2012. 
Tableau 1 : Poulies à demi-caisse (les dimensions sont en $\mathrm{cm}$ ).

\begin{tabular}{|c|c|c|c|c|c|c|}
\hline N. Cat & $\begin{array}{l}N^{\circ} \text { inv. } \\
\text { fouille }\end{array}$ & Localisation & État de conservation & Bois & Dimensions & Fig. \\
\hline 1 & $\begin{array}{l}7123 \\
7124 \\
7122\end{array}$ & $\begin{array}{l}1976 \\
\text { sur la coque }\end{array}$ & En trois fragments (deux demi-caisses et le réa) & $\begin{array}{c}\text { Chêne } \\
\text { vert }\end{array}$ & $\begin{array}{c}\text { Caisse : h. } 29 ; \text { I. } 12,5 \text {; ép. } 6,5 \text {; } \\
\text { diam. mortaises cylindriques } 5 . \text { L. évidement } \\
\text { central } 16 \\
\text { Réa : diam. } 8 \text {; ép. } 5 \text {; ergots : I. } 2 \text {; } \\
\text { diam. } 3 \text { à } 4\end{array}$ & $\begin{array}{l}1,1 \\
\text { et } 2\end{array}$ \\
\hline 2 & 5518 & $\begin{array}{c}1976 \\
\text { sur la coque }\end{array}$ & $\begin{array}{l}\text { Fragment de caisse avec un réa de poulie } \\
\text { à ergots tronconiques }\end{array}$ & Orme & $\begin{array}{l}\text { Réa : diam.7 ; ép. } 4 \text {; ergots : I. } 1,7 \text {; } \\
\text { diam. } 2,5 \text { à } 3,5\end{array}$ & \\
\hline 3 & 7888 & $\begin{array}{c}1976 \\
\text { sans localisation }\end{array}$ & $\begin{array}{c}\text { Fragment de caisse correspondant à la mortaise de l'essieu. } \\
\text { Une cheville traverse la demi-caisse dans sa largeur }\end{array}$ & & $\begin{array}{l}\text { Caisse : h. cons. } 11 \text {; ép. } 4 \text {; } \\
\text { diam. mortaise essieu } 2,5\end{array}$ & \\
\hline 4 & 7882 & $\begin{array}{c}1976 \\
\text { sur la coque }\end{array}$ & Réa à ergots tronconique & & $\begin{array}{c}\text { Réa : diam. } 7 \text {; ép. } 4 \text {; ergots : I. 1,7; } \\
\text { diam. } 2,5 \text { à } 3,5\end{array}$ & \\
\hline 5 & 8616 & 1977 & Fragment de partie supérieure d'une demi-caisse de poulie & & Caisse : I. max. conservée 6 ; épaisseur 7. & \\
\hline 6 & 11040 & $\begin{array}{l}1979 \\
\text { dans le puits } \\
\text { de la pompe } \\
\text { de cale }\end{array}$ & $\begin{array}{l}\text { Demi-caisse entière, bien conservée. Une cheville à chaque } \\
\text { extrémité assemble les deux moitiés. La partie latérale porte } \\
\text { une profonde engoujure }\end{array}$ & & $\begin{array}{l}\text { Caisse : h. tot. } 39,5 \text {; I. } 8 \text {; ép. } 3,7 \\
\text { diam. mortaise essieu : } 6,5 \\
\text { L. évidement central } 17,5\end{array}$ & 1,4 \\
\hline 7 & 11101 & $\begin{array}{l}1979 \\
\text { dans le puits } \\
\text { de la pompe } \\
\text { de cale }\end{array}$ & $\begin{array}{l}\text { Moitié supérieure d'une demi-caisse de poulie. Une cheville } \\
\text { relie les deux demi-caisses. Une mortaise pratiquée dans } \\
\text { l'épaisseur permet le passage du bout servant à la ligature } \\
\text { de l'estrope }\end{array}$ & & $\begin{array}{l}\text { Caisse : h. tot. conservée } 15 ; \text { I. env. } 8, \text { h. } \\
\text { restituable de la mortaise pour l'essieu } 7\end{array}$ & \\
\hline 8 & $\begin{array}{l}12597 \\
12609\end{array}$ & $\begin{array}{c}1980 \\
\text { à l'extérieur } \\
\text { de la coque, au } \\
\text { niveau de la quille }\end{array}$ & $\begin{array}{l}\text { Poulie entière mais en très mauvais état de conservation. } \\
\text { Demi-caisses assemblées dans la partie supérieure } \\
\text { par une cheville. Deux autres trous circulaires dans le haut } \\
\text { de la poulie pour la ligature de l'estrope }\end{array}$ & & $\begin{array}{l}\text { Caisse : h. } 28 ; \text { I. } 16 \text {; ép. } 5,5 \text {; } \\
\text { L. évidement central env. } 14 \\
\text { Réa : envergure totale } 10,2 \text {; ép. } 5,4 \text {, diam. } \\
\text { entre } 6,2 \text { et } 7,5 \text {; diam. ergots } 3,3 ; \text { I. } 2,4\end{array}$ & \\
\hline 9 & 14226 & $\begin{array}{c}1982 \\
\text { sous la coque, } \\
\text { à l'occasion } \\
\text { du prélèvement } \\
\text { de la quille }\end{array}$ & $\begin{array}{c}\text { Poulie presque entière formée de deux demi-caisses } \\
\text { assemblées et réa à ergots. Les deux demi-caisses sont } \\
\text { assemblées par une cheville à chaque extrémité. } \\
\text { Le haut de la poulie porte deux petits trous } \\
\text { qui permettaient de ligaturer l'estrope }\end{array}$ & & $\begin{array}{l}\text { Caisse : h. } 28,5 \text {; I. max } 17 \text {; ép. } 5 . \\
\text { Réa : envergure totale } 10,4 \text {; ép. } 5,4 \text {; } \\
\text { diam. entre } 6,3 \text { et } 7 \text {; diam. ergots } 4,2\end{array}$ & 1,3 \\
\hline
\end{tabular}

La présentation des pièces dans les tableaux suit l'ordre chronologique des campagnes de façon à respecter les contextes de découverte $^{2}$. En 1976 et 1977 la fouille a été particulièrement riche en pièces de gréement qui proviennent toutes de l'extrémité arrière de la coque, située au-delà des dernières amphores en place. Les documents iconographiques montrent en effet que les manœuvres de la voilure principale étaient regroupées à la poupe et il pouvait exister, à l'extrémité de la cale, des compartiments destinés aux rangements des agrès de rechange. En 1978, le nombre des pièces a considérablement diminué à mesure que l'on a quitté la zone arrière où étaient disposés la cabine et les rangements. En 1979, la plupart des objets proviennent du puits de la pompe de cale, situé à l'arrière du navire. La campagne 1980 a apporté peu d'éléments car elle a concerné l'avant. En 1982, les seuls objets découverts l'ont été sous la coque à l'occasion du prélèvement d'une section de la quille.

\section{LES PIÈCES DU GRÉEMENT}

\section{LES POULIES}

On connaît sur les navires antiques des poulies simples et des poulies plus complexes à réas multiples (infra) généralement en

2. Les descriptions qui suivent empruntent beaucoup aux rapports de fouille de P. Pomey pour les années 1976, 1977 et 1978 ; en 1979, 1980, 1982, rapports de fouille M.-B. Carre. L'ensemble de la documentation, reprise dans Carre 1983, est inédite. Pour des raisons contingentes, il n'a pas été possible de reproduire des photos à partir des négatifs ; je remercie chaleureusement mes relecteurs et Vincent Dumas qui a bien voulu mettre au net les dessins. bois, mais aussi parfois en métal. Seules des poulies simples en bois ont été trouvées durant les campagnes de 1976 à 1982 sur l'épave de la Madrague de Giens. Elles se rattachent à deux types différents qui ont coexisté pendant plusieurs siècles sans que l'on ait pu déceler une différence d'utilisation, les poulies à demi-caisses assemblées et les poulies monoxyles.

\section{Poulie à demi-caisses et réa à ergots}

(fig. 1, 1-4, tableau 1)

Ces poulies sont de forme générale ovoïde, avec un réa monoxyle muni de deux ergots tronconiques qui tourne dans un logement prévu à cet effet dans la face interne de la joue de chaque demi-caisse ${ }^{3}$. L'assemblage des deux demi-caisses est assuré par deux chevilles parallèles à l'axe du réa, qui traversent l'épaisseur des deux demi-caisses à chaque extrémité de la poulie. Une des poulies de la Madrague $\left(n^{\circ} 8\right)$ ne possède que celle de la partie supérieure, sans que l'on en saisisse le motif : il peut s'agir d'un simple oubli lors du façonnage. En revanche, il semble bien que deux chevilles aient assemblé la partie inférieure d'une poulie de l'épave de Kyrenia (Swiny, Katzev 1973, p. 351, fig. 12-13), mais l'on ne sait si cette pratique est occasionnelle ou récurrente dans les poulies d'époque classique. Une estrope, courant dans l'engoujure latérale, renforce la solidité de l'ensemble et sert à la suspension dans les cordages. La cannelure est très importante, puisqu'elle peut être creusée sur près de $3 \mathrm{~cm}$ $\left(n^{\circ} 9\right.$, fig. 1,3). Les deux petits trous qui apparaissent au sommet servaient à bloquer la ligature de l'estrope : on peut en voir un exemple très bien conservé sur un cap-de-mouton retrouvé sur

3. Une poulie à demi-caisses est illustrée dans Pomey 1997, p. 108. 
Tableau 2 : Poulies monoxyles (les dimensions sont en $\mathrm{cm}$ ).

\begin{tabular}{|c|c|c|c|c|c|c|}
\hline N. Cat & $\begin{array}{l}\mathrm{N}^{\circ} \text { inv. } \\
\text { fouille }\end{array}$ & Localisation & État de conservation & Bois & Dimensions & Fig. \\
\hline 10 & 7902 & 1976 & Réa à essieu mobile & & diam. 34 ; ép. 6,5 ; diam. essieu 6,4 & 1,5 \\
\hline 11 & & 1976 & Réa à essieu mobile & & diam. 6 , ép. 3,5 ; diam. essieu 2,3 & \\
\hline 12 & 8921 & 1977 & $\begin{array}{l}\text { Poulie dont la partie inférieure est détruite, portant un réa } \\
\text { à axe mobile. La partie supérieure de la caisse, étranglée, est } \\
\text { percée selon un axe parallèle à l'essieu du réa d'une grande } \\
\text { mortaise destinée au passage d'une estrope de grande } \\
\text { dimension }\end{array}$ & & $\begin{array}{c}\text { Caisse : L. cons } 21,5 \text {; I. } 8,5 \text {; ép. } 7,4 \\
\text { Réa diam. } 7,6 \text {; ép. } 4 \text {; diam. essieu } 2 \text {; diam. } \\
\text { de la mortaise } 4,2\end{array}$ & 1,6 \\
\hline 13 & 8737 & 1977 & $\begin{array}{c}\text { Partie supérieure de caisse. La mortaise de l'essieu du réa } \\
\text { disparu est en partie conservée. Il ne devait pas y avoir ici } \\
\text { de trou pour la suspension, mais l'estrope était logée dans } \\
\text { une engoujure creusée sur les parties latérales de la caisse } \\
\text { deux trous cylindriques étaient percés dans l'épaisseur } \\
\text { de la tête pour la ligaturer. Des restes de cordage étaient } \\
\text { présents dans l'un de ces trous }\end{array}$ & Orme & $\begin{array}{l}\text { L. cons } 0,19 \text {; I. max. restituée } 0,8 \text {; ép. } 0,7 \text {; } \\
\text { diam. de la mortaise pour l'essieu 0,015 à } \\
0,020 \text {; diam. des trous de ligature } 0,014\end{array}$ & \\
\hline 14 & 8615 & 1977 & Réa de poulie avec un fragment d'axe conservé & & diam. 9 ; ép. 5,5 ; diam. essieu 4,6 & \\
\hline
\end{tabular}

l'épave Laurons 2 (Ximénès, Moerman 1990, p. 8, fig. 3) : deux petits cordages passant dans les trous supérieurs assurent la bridure de l'estrope. Les réas ont la forme d'un cylindre avec un creux très marqué pour le passage du cordage. Ils sont solidaires de leur axe et tournent dans les mortaises des demi-caisses. Leur décalage vers le bas de la poulie permet au cordage dont la poulie devait modifier la direction de courir librement au travers de la caisse sans risque de frottement.

Outre les trois poulies en plus ou moins bon état trouvées avec leurs réas, on compte des éléments ayant appartenu à des objets différents : deux portions de caisses ( $\mathrm{n}^{\circ} 6$ et 7 ) dont le diamètre de la mortaise de l'essieu du réa est respectivement de 6,5 et $7 \mathrm{~cm}$; et deux réas isolés ( $\mathrm{n}^{\circ} 2$ et 4 ) dont le diamètre de l'essieu est compris entre 2,5 et $3,5 \mathrm{~cm}$. À l'exception des deux pièces tombées dans le puits de la pompe de cale, on observera la cohérence de leur découverte à l'arrière, sur les parties hautes conservées de la coque $\left(n^{\circ} 1,2,4\right)$ ou tombées sous le flanc du navire $\left(n^{\circ} 8\right.$ et 9$)$. Les trois poulies entières ( $n^{\circ} 1,8$ et 9$)$ présentent des dimensions tout à fait comparables; seule la poulie $\mathrm{n}^{\circ} 6$, avoisinant $40 \mathrm{~cm}$ de haut, est exceptionnellement grande.

Ce type de poulie a disparu dans la marine moderne, mais est très régulièrement présent sur les épaves antiques. La plus ancienne est attestée dès le $\mathrm{IV}^{\mathrm{e}} \mathrm{S}$. av. J.-C sur le navire de Kyrenia (Swiny, Katzev 1973, p. 351, fig. 12 et 13). On les retrouve ensuite en Méditerranée avec une prédominance dans les deux derniers siècles de la République ${ }^{4}$. L'exemple le plus récent est longtemps resté celui des épaves du lac de Nemi datées du règne de Caligula. Cependant il n'avait pas attiré l'attention des chercheurs car les divers éléments en avaient été replacés dans une «plate-forme sur rouleaux » (Ucelli 1950, p. 193-195, fig. 213-214), en réalité une reconstitution abusive avec des parties de poulies à demicaisses, parfaitement identifiables sur la photographie et le dessin. On voit bien dans la reconstruction comment la forme des réas est forcée pour obtenir des « rouleaux » tronconiques corroborant l'hypothèse émise par le fouilleur ${ }^{5}$. Une poulie entière, très bien conservée, a ensuite été trouvée à Caesarea

4. On en trouvera la liste très complète, ainsi que celle des poulies monoxyles simples ou multiples, dans l'article de Oleson 1983, à compléter avec Carre, Rival 1988, p. 113-115 ; Ximénès, Moerman, 1990, p. 5-6 ; Cornelio Cassai 1990, p. 43, p. 281-283 (n 284-285) et les autres références citées ici.

5. Sur la réinterprétation d'autres pièces des épaves de Nemi, voir Carre 2007, en particulier p. 55 .
Maritima dans un contexte portuaire du $\mathrm{I}^{\mathrm{er}}$ s. ap. J.-C. (Oleson 1983). Mais l'attestation la plus fiable de la continuité de l'usage de ce type est fournie par l'épave Grado 1, très bien datée du milieu du $\mathrm{II}^{\mathrm{e}} \mathrm{s}$., où une poulie à demi-caisse a été trouvée sous la coque (Beltrame, Gaddi 2005, p. 80, fig. 2). Elle est contemporaine d'un réa monoxyle provenant de fouilles dans le port de Myos Hormos en mer Rouge (Whitewright 2007, p. 284, fig. 3, WO270). Il ne semble plus y en avoir au-delà du $\mathrm{II}^{\mathrm{e}}$ s., mais les attestations sont si peu nombreuses qu'il est possible que de nouvelles découvertes viennent infirmer cette impression.

\section{Poulie simple monoxyle (fig. 1, 5-6, tab. 2)}

Le navire de Giens était équipé avec un autre type de poulie simple, à caisse monoxyle munie d'un réa dont l'axe est mobile. Ces objets présentent une variété d'aspect plus grande que le type précédent. Cependant des caractéristiques communes peuvent être dégagées : ils sont composés de trois éléments indépendants : la caisse, l'essieu et le réa. La caisse, fabriquée dans une seule pièce de bois, est percée de plusieurs mortaises : dans sa partie supérieure, une mortaise cylindrique ménage le passage d'un essieu autour duquel tourne le réa. Celui-ci est un disque de bois rond percé d'un trou central pour le passage de l'essieu. Le cordage est guidé dans sa course autour du réa par la forte engoujure de la partie latérale. Une deuxième mortaise, plus grande, est pratiquée dans la caisse pour ménager le logement du réa. Ces poulies sont également percées dans leur partie inférieure d'une mortaise circulaire permettant d'y passer un cordage courant, ou bien encore une estrope pour suspendre ou tourner la poulie à un endroit bien spécifique. Seules deux poulies en relativement bon état de conservation ont été récupérées à la Madrague de Giens.

Les réas isolés sont placés à la fin du tableau car ils pourraient également appartenir à une autre catégorie, celle des poulies multiples, bien qu'aucun élément n'aille dans ce sens sur cette épave. Au demeurant, le plus ancien témoignage archéologique est plus tardif que le naufrage du navire de Giens, ce qui pourrait expliquer son absence ici : il s'agit de la poulie à trois doubles réas superposés de Port-Vendres 2, qui a sombré avant le milieu du I ${ }^{\text {er }}$ S. ap. J.-C. (Colls et al. 1977, p. 10 et fig. 2). Des palans à réas multiples figurent aussi sur l'arc d'Orange, daté du règne de Tibère (fig. 1,7). C'est encore à Port-Vendres, sur l'épave 1 datée de la fin du IV ${ }^{\mathrm{e}} \mathrm{s}$. au plus tôt, que l'on trouve la poulie de bois la plus complexe, puisqu'elle porte deux étages 

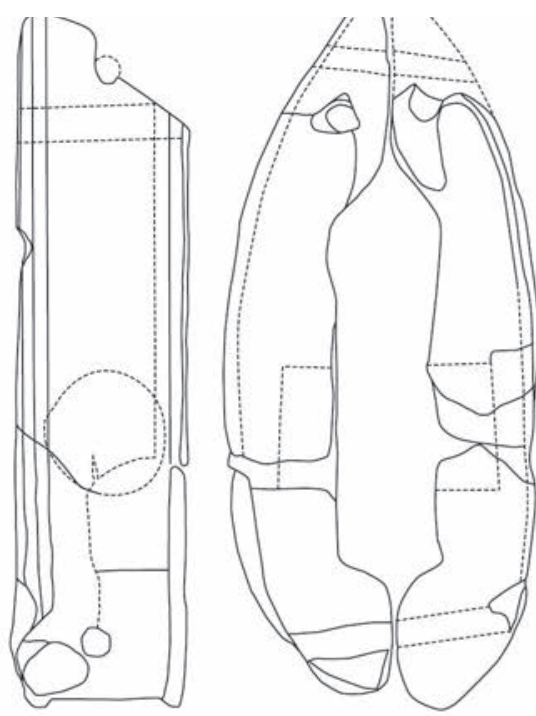

1

0

$5 \mathrm{~cm}$
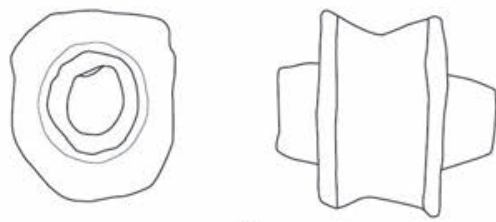

2
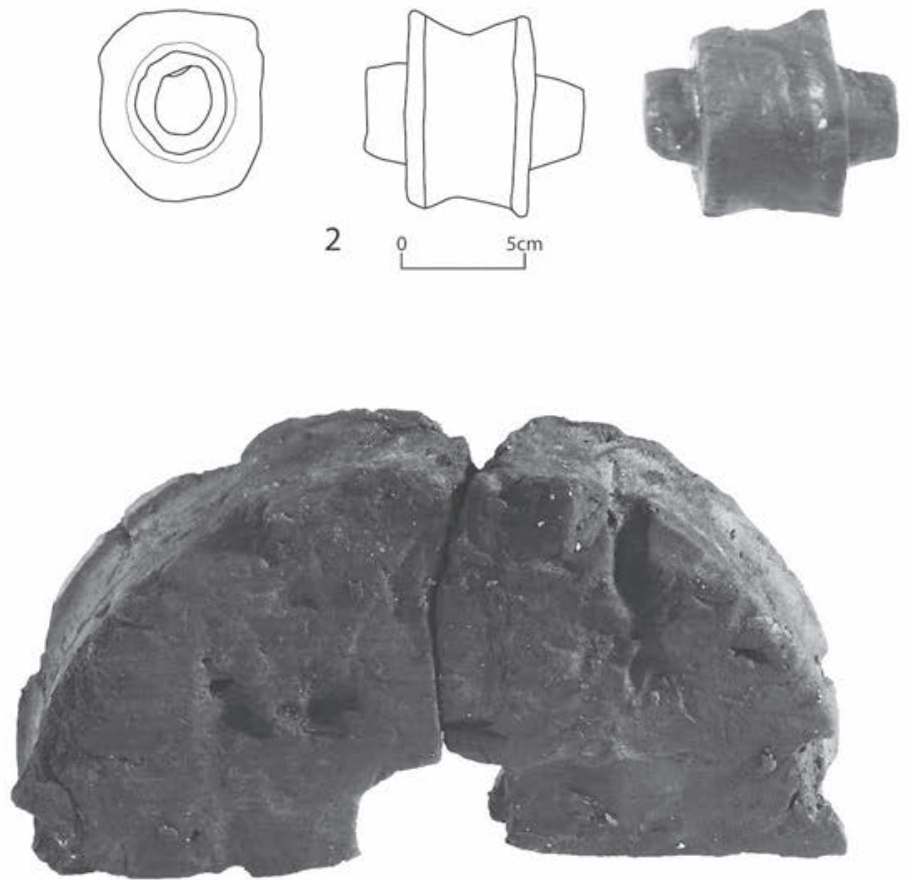

5

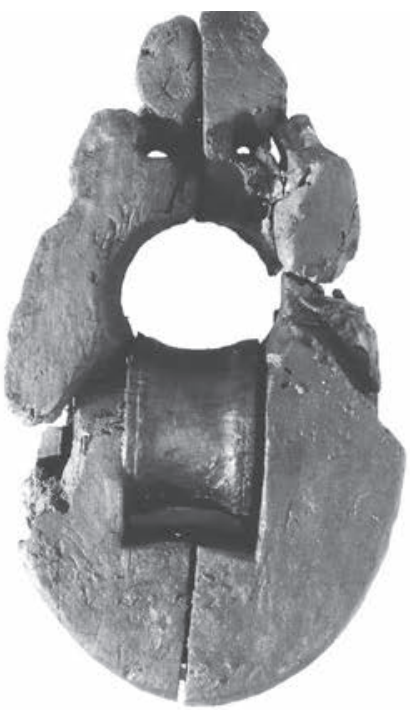

3
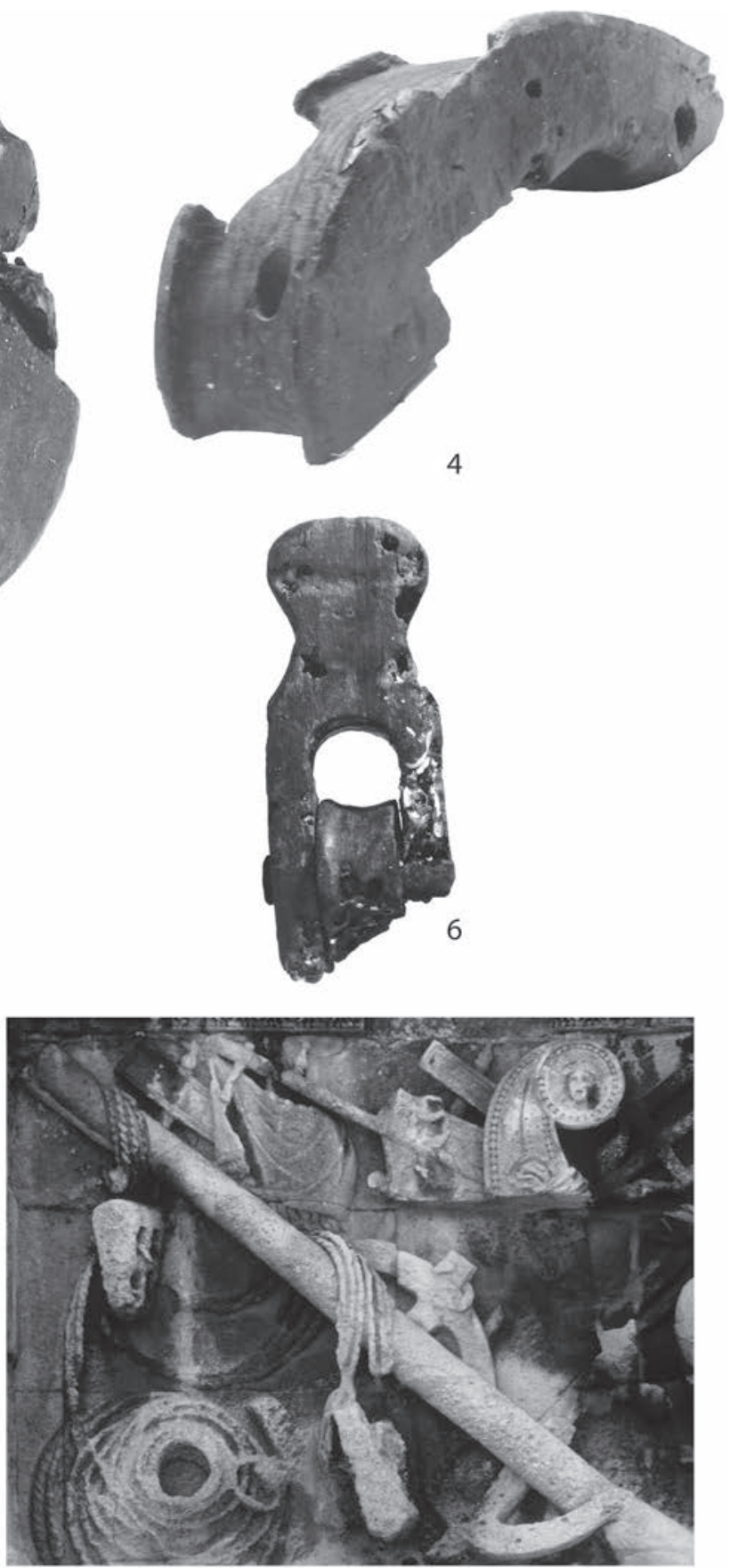

7

Fig. 1 : Poulies à demi-caisses (1-4) et monoxyles (5-6). Dessins M. Rival (CNRS, CCJ), V. Dumas (AMU, CNRS, CCJ); clichés A. Chéné, Ph. Foliot (CNRS, CCJ). Les photographies ne sont pas à l'échelle. Arc d'Orange, panneau des dépouilles navales (7). Cliché A. Chéné, Ph. Foliot.

de réas perpendiculaires, respectivement de 5 et 4 réas (Carre 1983, p. 45), mais d'autres poulies à plusieurs réas sont connues pour l'époque romaine (Oleson 1983 ; Beltrame, Gaddi 2005).

Parmi les pièces récupérées à la Madrague de Giens, les deux poulies en bon état sont différentes : le cordage de suspension de la poulie $\mathrm{n}^{\circ} 12$ passait dans une mortaise ménagée dans la tête, tandis que la poulie $\mathrm{n}^{\circ} 13$ était suspendue au moyen d'un cordage logé dans l'engoujure latérale, comme les poulies à demi-caisses. Toute tentative de préciser davantage l'utilisation et la localisation de ces deux poulies dans le gréement s'avère impossible, car elles peuvent être placées à une infinité d'endroits dans la mâture. Cependant, la présence à proximité de la poulie $n^{\circ} 12$ d'un anneau de cargue ( $\mathrm{n}^{\circ} 25$, inv. 8918 ) pourrait évoquer son utilisation pour la manœuvre de la voilure.

Parmi les réas isolés, non rattachables à une poulie en particulier, le $\mathrm{n}^{\circ} 10$ frappe par ses dimensions hors normes. On peut essayer de calculer le poids qu'une poulie de cette taille pouvait soulever en suivant les indications données dans les traités de la marine moderne, puisque l'épaisseur du réa des poulies monoxyles est fonction de celle du cordage et que les 
matériaux utilisés pour les cordages sont restés les mêmes depuis l'Antiquité. Les analyses des cordages retrouvés sur l'épave de la Madrague ${ }^{6}$ confirment qu'il s'agit de chanvre, considéré encore à l'époque moderne comme le meilleur matériau pour cet usage. Cette poulie pouvait théoriquement soulever un poids en mouvement de plus de 3 tonnes $^{7}$ et n'est dépassée en taille que par celle trouvée dans le lac de Nemi (Ucelli 1950, p. 181-182, fig. 197), dont le diamètre du réa est d'environ $50 \mathrm{~cm}$ et l'épaisseur de $10 \mathrm{~cm}$. Haute de $1,08 \mathrm{~m}$, elle était renforcée de barres de fer et sa caisse n'était pas refermée au-dessus du réa. La poulie B17 du Grand Ribaud D, dont la charge de rupture en mouvement a été calculée à $1518 \mathrm{~kg}$, est également une poulie ouverte (Carre, Rival 1988, p. 114). Il est possible que la poulie de la Madrague de Giens ait appartenu à cette catégorie, qui n'est plus attestée dans la marine moderne, car l'ouverture des poulies coupées se situe sur la joue et non à une extrémité de la caisse (Bonnefoux, Pâris 1856, s.v. Coupée (poulie). On distingue, sur le sarcophage de Naevoleia Tyche à Pompéi (Pomey 1997, p. 40), une poulie fixée au pied du grand mât probablement destinée à la manœuvre de la vergue : c'est peut-être à cet usage qu'était destinée la poulie de la Madrague.

La découverte des poulies des deux types sur l'épave n'a pas contribué à éclaircir la question de leur différence éventuelle de fonction. Les fragments d'une poulie à demi-caisse $\mathrm{n}^{\circ} 5$ et un réa de poulie monoxyle $n^{\circ} 14$ ont été retrouvés au même endroit, ce qui pourrait évoquer leur utilisation dans un palan. Les poulies à demi-caisses et monoxyles sont en effet présentes simultanément sur un grand nombre d'épaves entre le $\mathrm{II}^{\mathrm{e}}$ s. av. J.-C. et le $\mathrm{II}^{\mathrm{e}}$ ap. J.-C. On constate cependant que, si le diamètre des réas de certaines poulies monoxyles ne dépasse pas celui des réas à ergots qui comptent entre 6 et $7 \mathrm{~cm}$, toutes les grandes poulies, dont le diamètre du réa est supérieur à $11 \mathrm{~cm}$, sont monoxyles. En outre, ce sont les seules à pouvoir porter plusieurs réas parallèles ou perpendiculaires les uns aux autres, répondant ainsi à des contraintes de renvois complexes. Cependant, certaines des poulies à demi-caisses de l'épave Madrague de Giens montrent des dimensions considérables (près de $40 \mathrm{~cm}$ de haut pour la poulie $n^{\circ} 6$ ). La position, et donc la fonction, des unes et des autres obéit à certaines règles qui nous échappent. L'iconographie n'est pas d'une grande aide sur ce point, car le type n'est pas identifiable précisément. En outre, la comparaison avec la marine à voile moderne est impossible puisque le type à demi-caisses a disparu précocement, démontrant sans doute ainsi une moins bonne adéquation que leurs homologues monoxyles aux nécessités de la navigation. Peut-être le point de faiblesse constitué par l'assemblage des deux demi-caisses a-t-il joué un rôle dans son abandon.

6. Inédit, analyses de H. Masurel. L'usage du chanvre est bien attesté par les sources anciennes : voir Casson 1971, p. 231. À Nemi les cordages sont également en chanvre : Ucelli 1950, p. 268.

7. Le réa mobile a une épaisseur égale à un tiers de la circonférence du cordage (Barrot de Gaillard 1939, p. 229). La charge de rupture d'un cordage en chanvre est donnée par la formule $\mathrm{R}=0,5 \mathrm{C}^{2}$ (Devillers 1971, p. 12). R est exprimé en $\mathrm{kg}$ et $\mathrm{C}$ en $\mathrm{mm}$. La poulie peut recevoir un cordage de $65 \mathrm{~mm} \times 3=$ $195 \mathrm{~mm}$ de circonférence. La charge de rupture est donc de $19012 \mathrm{~kg}$. La charge de sécurité d'un cordage ne doit pas dépasser le $1 / 5^{\mathrm{e}}$ de la charge de rupture lorsque le cordage est immobile (= $3802 \mathrm{~kg}$ ), le $1 / 6^{\mathrm{e}}$ s'il est en mouvement (= $3168 \mathrm{~kg})$, le 1/10e s'il est soumis à des à-coups (=1901kg).
MOQUES (fig. 2, tab. 3)

Les moques et caps-de-mouton appartiennent à la même famille et les deux types sont communs sur les bateaux antiques $^{8}$. La forme de ces objets est plutôt homogène ${ }^{9}$, puisqu'il s'agit dans les deux cas de pièces de bois plates, ovoïdes, percés d'un trou pour les moques ou de plusieurs trous pour les caps-de-mouton circulaires et munies d'une engoujure sur leur périphérie. À l'époque moderne, les deux séries de pièces étaient utilisées comme des sortes de poulies sans réa pour raidir les manœuvres dormantes, principalement les étais et les haubans, et elles étaient disposées par paire : une ride passant par le ou les trous centraux sert à opérer la tension. Elle fait dormant sur un nœud de ride, puis passe alternativement dans l'un, puis dans l'autre cap-de-mouton. Les haubans ou autres manœuvres sont alors raidies au palan, le mou de la ride étant repris à mesure jusqu'à obtenir la tension désirée (Devillers 1971, p. 120-121). L'engoujure sert à recevoir la manœuvre supérieure.

A la Madrague de Giens, pour autant que l'on puisse en juger par l'état de conservation des pièces, seules des moques à un seul trou ont été retrouvées. De forme générale ovoïde, elles sont percées dans leur extrémité supérieure, du côté de la pointe, d'un petit trou (diam. entre 1 et $1,7 \mathrm{~cm}$ ) et dans la partie la plus large d'un grand évidement circulaire (diam. entre 3 et $5 \mathrm{~cm}$ ).

La collection comprend cinq moques entières ou de forme complète, cinq fragments et une moque de plus petites dimensions. Seules deux années de fouille ont livré ces objets : 1976, lors de l'exploration de l'extrémité arrière de la coque, riche en pièces de gréement ( $\left.{ }^{\circ} 15-20\right)$, et 1979 où les pièces ont été retrouvées dans le puits de la pompe de cale ( $\left.n^{\circ} 21-24\right)$.

Les dimensions de quatre des cinq objets entiers sont identiques, $16 \mathrm{~cm}$ de haut, ce qui semble correspondre à la hauteur restituable des pièces cassées. Une seule est de dimension nettement inférieure $\left(\mathrm{n}^{\circ} 25\right)$. Les engoujures périphériques sont particulièrement profondes. Le petit trou supérieur devait servir à la ligature du cordage principal, comme le montre l'exemple déjà évoqué sur les Laurons 2 (Ximenès, Moerman, 1990, p. 8 et fig. 3 ; Pomey 1997, p. 108).

Les traités modernes mentionnent l'utilisation de moques pour les étais, mais les pièces antiques, avec leur épaisseur autour de $2 \mathrm{~cm}$, ne peuvent garnir que des cordages formant une boucle de $6 \mathrm{~cm}$ de circonférence au maximum, c'est-à-dire moins de $2 \mathrm{~cm}$ de diamètre. Il est intéressant de constater que ces objets étaient encore groupés lors de leur découverte, en particulier les deux moques $\mathrm{n}^{\circ} 17$, retrouvées superposées et avec la poulie $n^{\circ} 1$. La localisation dans la partie arrière pourrait correspondre à leur fonction dans les haubans, qui est de maintenir le mât par le travers et par l'arrière.

Il est bien difficile, vu le nombre de restes, de décider si la forme de ces pièces a connu une évolution au cours du temps : on constate cependant que, sur les épaves républicaines Chrétienne $C$ datée du deuxième quart du $\mathrm{II}^{\mathrm{e}}$ s. av. J.-C. ${ }^{10}$,

8. Mais peut-être pas à la même période : voir infra.

9. «Moque : Bloc de bois à peu près lenticulaire, cannelé sur l'épaisseur de son contour pour recevoir une estrope et évidé dans sa partie centrale » (Bonnefoux et Pâris, s.v. moque). « Caps-de-mouton ; forte lentille en bois percée de trois trous. Sur leur sens circulaire, les caps-de-mouton ont une engoujure » (id., s.v. cap de mouton)

10. Deux moques de 4,5 et $5,3 \mathrm{~cm}$ de haut, pour un diamètre de l'évidement central de 1,7 et $2,2 \mathrm{~cm}$. 

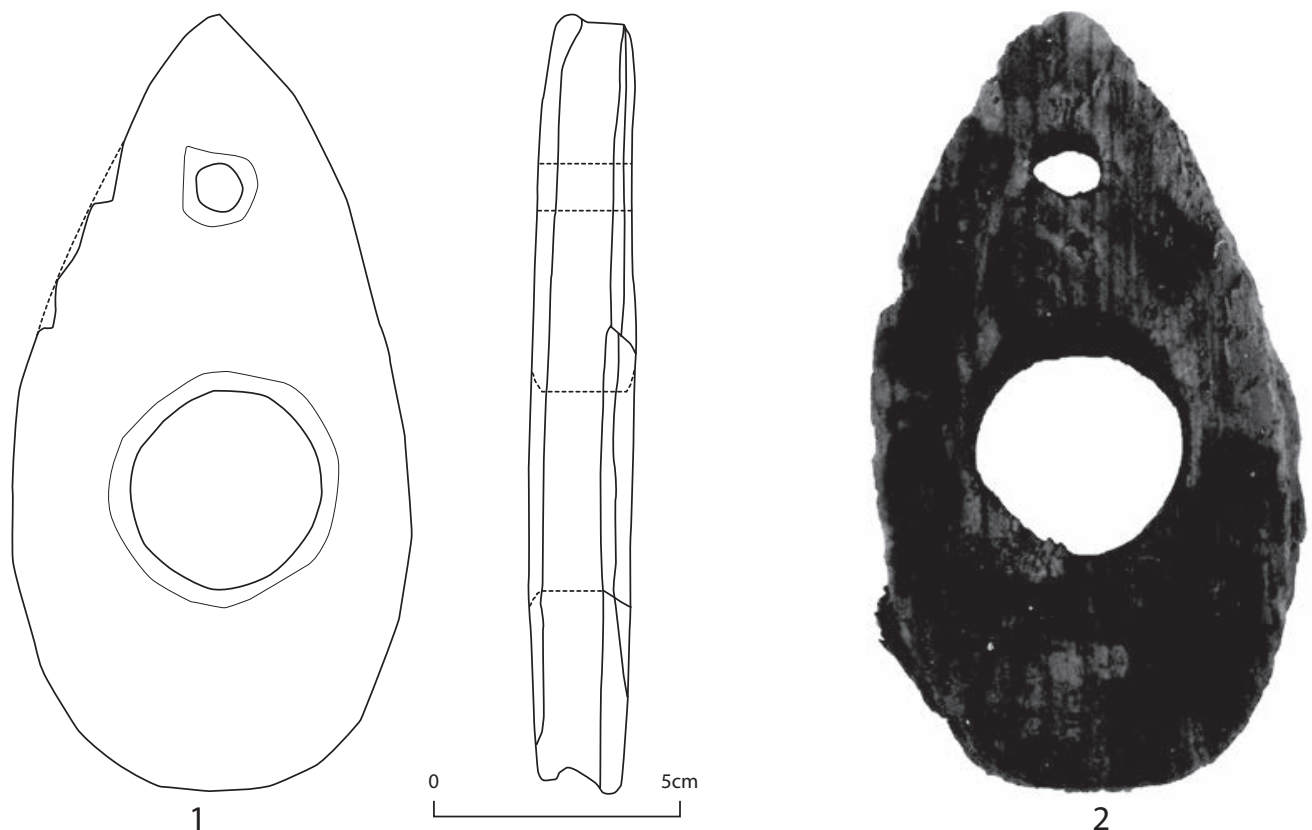

Fig. 2 : Moques. Dessins M. Rival, V. Dumas ; clichés A. Chéné, Ph. Foliot. Les photographies ne sont pas à l'échelle.

Tableau 3 : Moques (les dimensions sont en $\mathrm{cm}$ ).

\begin{tabular}{|c|c|c|c|c|c|}
\hline N. Cat & $\begin{array}{l}N^{\circ} \text { inv. } \\
\text { fouille }\end{array}$ & Localisation & État de conservation & Dimensions & Figure \\
\hline 15 & 6626 & 1976 & Entière & h. 16 ; I. 8,5 ; ép. 2 & 2,1 \\
\hline 16 & 7034 & 1976 & Partie inférieure & h. cons. 13,9 ; I. 8,3 ; ép. 1,8 ; diam. trou principal 3,1 & \\
\hline 17 & 7120 & 1976 & 2 objets, un entier et l'autre fragmentaire & h. 16 ; I. 8,5 ; ép. 2 & \\
\hline 18 & 7862 & 1976 & Partie supérieure & & \\
\hline 19 & 7877 & 1976 & Fragment & & \\
\hline 20 & 7878 & 1976 & Cassée, mais forme archéologiquement complète & & \\
\hline 21 & 11030 & $\begin{array}{c}1979 \\
\text { puits de la pompe }\end{array}$ & Entière & $\begin{array}{l}\text { h. } 16 ; \text { I. } 7,5 \text {; ép. } 2,1 \text {; diam. trou principal } 5 \text {; diam. trou } \\
\text { secondaire } 1,1\end{array}$ & \\
\hline 22 & 11031 & $\begin{array}{c}1979 \\
\text { puits de la pompe }\end{array}$ & Entière & $\begin{array}{l}\text { h. } 16 ; \text { I. } 8,5 \text {; ép. } 2,4 \text {; diam. trou principal } 4,5 \text {; diam. trou } \\
\text { secondaire } 1,6\end{array}$ & 2,2 \\
\hline 23 & 11032 & $\begin{array}{c}1979 \\
\text { puits de la pompe }\end{array}$ & Partie supérieure & h. cons. 9,5 ; I. 6,5 ; ép. 2,2 ; diam. trou secondaire 1,7 & \\
\hline 24 & 11107 & $\begin{array}{l}1979 \\
\text { puits de la pompe }\end{array}$ & Moque entière & $\begin{array}{l}\text { h. } 11 ; \text { I. } 7,5 \text {; ép. } 1,8 \text {; diam. trou principal } 4 \text {; diam. trou } \\
\text { secondaire } 1\end{array}$ & \\
\hline
\end{tabular}

Madrague de Giens et Fos 1, du I ${ }^{\mathrm{er}}$ s. av. J.-C., seules des moques ont été repérées ${ }^{11}$. Le témoignage le plus tardif, vers 10 av. J.-C., est celui de Comacchio (Cornelio Cassai 1990, p. 43, 281-283, $\mathrm{n}^{\circ}$ 286-287), tandis que les caps-de-mouton à 2 ou 3 trous sont présents au $\mathrm{II}^{\mathrm{e}}$ s. ap. J.-C. sur les épaves Laurons 2, Grado 1 et à Myos Hormos ${ }^{12}$. L'iconographie, qui atteste la présence de ces pièces, montre seulement qu'elles étaient disposées par paire, comme sur le bas-relief de la collection Torlonia où l'on en distingue quatre paires fixées sur les haubans latéraux (Pomey 1997, p. 82).

11. Chrétienne $C$ : Joncheray 1975 , p. 104 et fig. 50,4 et 5 ; Fos 1 : inédit (rapport de fouille M.-P. Jézégou, S. Pincemin, C. Vignacq 1980)

12. Des caps-de-mouton à deux ou trois trous principaux disposés en triangle sur les épaves Laurons 2 (Ximénès, Moerman 1990, p. 5-9) et Grado (Beltrame, Gaddi 2005, p. 80, fig. 1), ou trois trous en ligne dans le port de Myos Hormos (Whitewright 2007, p. 284, fig. 2).

\section{ANNEAUX ET CONDUITS DE MANOEUVRE}

Un certain nombre de pièces annulaires en bois a pu être attribué au système du chapelet de la pompe de cale sur les épaves (Carre, Jézégou 1984). C'est le cas, à la Madrague de Giens, de dix anneaux à bords abattus, munis ou non de trous secondaires (Carre 2007, p. 60 et fig. 6,2). D'autres pièces trouvées sur l'épave sont de plus difficile identification. Les trois séries de pièces décrites ci-dessous sont à placer dans la catégorie des agrès de voile, anneaux ou conduits de manœuvre.

25. Trois anneaux entiers et cinq fragments en forme de languette, taillés dans une pièce de bois plate dont la partie la plus large est percée de deux trous. Une gorge entaille la face inférieure (inv. 6125, 6459, 7617, 7301, 7876, 8918) (fig. 3,1).

L. 8,5 à $8,8 \mathrm{~cm}$; h. 6,9 à $7,5 \mathrm{~cm}$; ép. 1 à $1,5 \mathrm{~cm}$; diam. int. 4 à $4,5 \mathrm{~cm}$; diam. trous secondaires $0,5 \mathrm{~cm}$. 


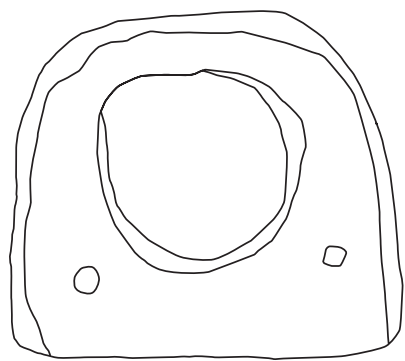

1

0

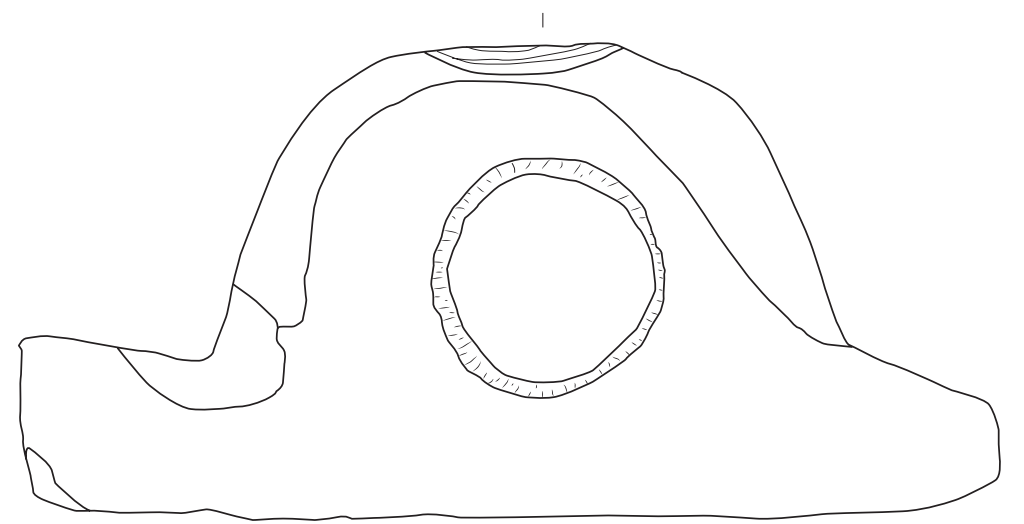

3

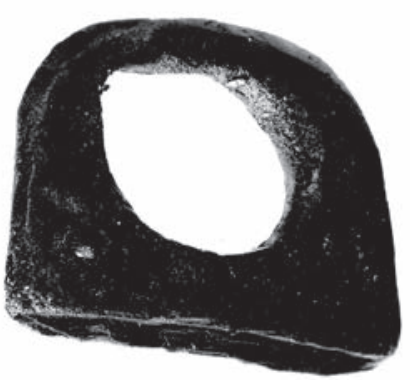

$\mathrm{cm}$

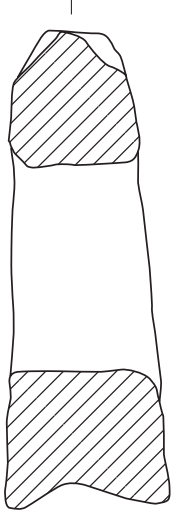
$5 \mathrm{~cm}$

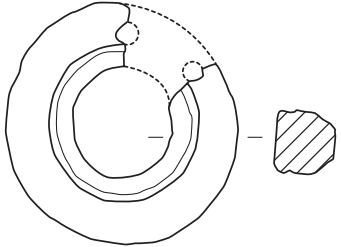

2 $5 \mathrm{~cm}$

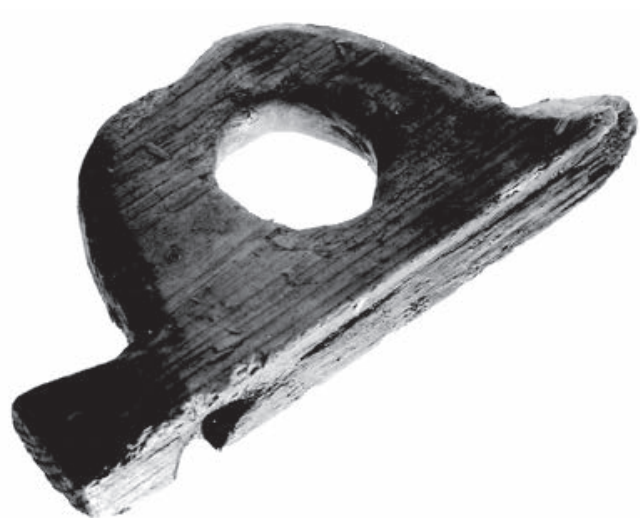

Fig. 3 : Anneaux de cargue et filoir. Dessins M. Rival, V. Dumas ; clichés A. Chéné, Ph. Foliot. Les photographies ne sont pas à l'échelle.

Assez rarement attestés (ou publiés), on trouve d'autres exemples de ces pièces sur la Chrétienne C (Joncheray 1975, p. 104, fig. 50,3) et Ladispoli (Carre 1993, p. 26, fig. 15), dont les hauteurs sont respectivement de 4,5 et $7,9 \mathrm{~cm}$, le diamètre intérieur de 2,7 et 3,6 cm.

26. Deux anneaux toriques complets comportant 2 petits trous côte à côte et un fragment appartenant peut-être à la même série (inv. 5844, 7879 et peut-être 7629) (fig. 3,2).

Diam. ext. $5 \mathrm{~cm}$; diam. int. 2,5 cm ; ép. $1,5 \mathrm{~cm}$; diam. trous secondaires $0,5 \mathrm{~cm}$.

Un anneau semblable a été trouvé sur l'épave Fos $1^{13}$. De nombreux exemples proviennent du port de Myos Hormos, où un anneau est encore ligaturé à un fragment de voile (Whitewright 2007, p. 286, fig. 4).

Ces deux séries de pièces sont à relier au système des cargues destinées à faire prendre à la voilure des configurations différentes ${ }^{14}$. L'engoujure que portent les languettes sous leur base servait à la fixation de l'anneau à un cordage ou une ralingue. Les anneaux de bois sont fixés sur la voile par des cordages passant par les trous secondaires, comme à Myos Hormos. À ces pièces on peut ajouter de simples anneaux toriques, très fré-

13. Inédit, rapport fouille M.-P. Jézégou 1981.

14. Pour le détail de la manœuvre des cargues sur la voile carrée voir Pomey 1997 , p. $80-82$. quents sur les épaves, qui devaient être cousus sur la voile ${ }^{15}$. Tous ces anneaux ont pu servir de filoirs de cargue. Celles-ci, passant séparément autour de la vergue, redescendent ensuite sur le pont en arrière du mât pour être tournées sur les râteliers, comme cela est bien visible sur la représentation du navire Europa à Pompéi (Pomey 1997, p. 83). Cependant, si ce dispositif est bien attesté dans l'iconographie, il n'est pas possible d'y distinguer l'utilisation des différents types.

Les anneaux de plomb, trouvés en grande quantité sur certaines épaves ${ }^{16}$, ont longtemps été identifiés comme des anneaux de cargue. Leur poids, la malléabilité du plomb, la mention de Pline $(N H, 13,18,62)$ qui signale pour les anneaux de cargue de l'utilisation d'un bois très dur, le $c u c i$, fruit du palmier doum, rendent cette proposition incertaine ${ }^{17}$. Cependant, la fonction des anneaux de plomb n'est pas identifiée précisément. L'hypothèse d'anneaux destinés à lester les filets de pêche n'est pas totalement satisfaisante, d'autant que, à la Madrague de Giens, ils ont été découverts à proximité immédiate des anneaux de bois.

15. Au moins un anneau complet, de $7,5 \mathrm{~cm}$ de diam. ext. et de $4 \mathrm{~cm}$ de diam. int., a été trouvé sur l'épave de Giens (fouille 1976, inv. 7300).

16. J. Whitewright (2007, p. 286) range les anneaux de bois et de plomb dans la même catégorie.

17. 37 anneaux de plomb trouvés à la Madrague de Giens, tous identiques (diam. ext. 6,5 à $7 \mathrm{~cm}$; diam. int. 4,5 cm ; ép. 0,6 à 0,9 cm.). 
27. Anneau monté sur une longue pièce de bois, munie d'ergots à ses extrémités. Une longue gorge entaille la face inférieure (Inv. 7861) (fig. 3,3).

L. $20 \mathrm{~cm}$; h. $10 \mathrm{~cm}$; ép. $3 \mathrm{~cm}$; diam. int. $4 \mathrm{~cm}$.

Cette pièce pourrait avoir été utilisée comme un conduit de manœuvre ou filoir ; «On appelle conduits de manœuvre des petites pommes, cosses ou margouillets engoujés latéralement pour être amarrés aux haubans et qui, étant percés de haut en bas, servent à diriger des cordages ou manœuvres venant du haut du gréement pour arriver jusqu'au pont ou à l'appel des poulies de retour »(Bonnefoux et Pâris, s.v. Conduits de manœuvre). La pièce de Giens est engoujée sur sa face inférieure, ce qui peut indiquer qu'elle était rousturée sur une manœuvre au moyen d'estropes passant sur ses ergots ; un autre cordage passait par son évidement central. Une pièce absolument identique, mais beaucoup plus petite $(4,5 \mathrm{~cm}$ de long) a été trouvée sur un navire portugais du XVIII ${ }^{\mathrm{e}} \mathrm{s}$. coulé à Monbasa au Kenya (Piercy 1977 18 ). Un objet similaire, à son tour de plus faible dimension (longueur $6 \mathrm{~cm}$; diam. trou central $0,4 \mathrm{~cm}$ ), était présent sur l'épave Grand Ribaud D (Carre, Rival 1988, p. 115, fig. XLV B8).

Le nombre de pièces de gréement est faible au regard de la dimension du navire de Giens, un des plus grands connus par l'archéologie qui, avec ses deux mâts et ses immenses voiles carrées, devait avoir fière allure avant son naufrage devant le port d'Olbia. La présence de deux poulies et de diverses autres pièces dans le puits de la pompe confirme l'intervention très rapide des urinatores (Tchernia 1988), puisqu'elles devaient se trouver encore sur le sommet du chargement, voire sur le pont qui a dû être démoli, s'il ne l'était pas déjà au moment du naufrage, pour permettre l'accès des plongeurs à la machinerie. Si ces derniers ont emporté, avec les amphores, la pompe de cale, nous privant ainsi de l'exemple d'une machine peut-être plus complexe que celles que nous connaissons par ailleurs, nous leur devons donc la préservation de quelques-unes des pièces de gréement tombées dans le puits béant au milieu de la coque avant la disparition complète de la mâture emportée par les vagues.

Les dimensions de certaines des pièces préservées (la poulie $\mathrm{n}^{\circ} 10$ par exemple, les moques, les anneaux de cargue) sont cohérentes avec les proportions du navire. Les pièces comparables, mais bien plus petites, provenant de la Chrétienne $C$ gréaient un navire de $18 \mathrm{~m}$ de long (Joncheray 1975, p. 64), que l'on peut mettre en perspective avec les 40 m estimés de la Madrague de Giens. Bien d'autres objets, parfois énigmatiques, ont été retrouvés. Ils concernent les pièces d'amarrage et de manœuvre des ancres et des voiles, les outils pour la confection des voiles et cordages. Leur étude, en lien avec la restitution et la publication finale, permettra de mieux appréhender le système technique de ce navire de taille exceptionnelle.

Marie-Brigitte CARRE Aix Marseille Univ, CNRS, Centre Camille Jullian, Aix-en-Provence, France marie-brigitte.carre@univ-amu.fr

\section{BIBLIOGRAPHIE}

\section{BARRot de GAILlARD \\ 1939 Construisez des modèles réduits de marine, Paris, éd. Barrot de Gaillard.}

Bonnefoux de P. M. J., PÂRIs F. E.

1856 Le Dictionnaire de la Marine à Voile, Paris (réimpression anastatique de la 2 e édition, Paris, René Baudoin éd., 1980).

Beltrame B., Gaddi D.

2005 The Rigging and the « Hydraulic System » of the Roman Wreck at Grado, Gorizia, Italy, IJNA, 34 .1, p. 79-87.

\section{Carre M.-B.}

1983 Gréement et équipement des navires gréco-romains d'après les documents archéologiques, thèse de $3^{\mathrm{e}}$ cycle sous la direction d'André Tchernia, Université de Provence, Aix-en-Provence, mars 1983 [non publiée].

1993 L'épave à dolia de Ladispoli (Étrurie méridionale). Etude des vestiges de la coque, Archaeonautica, 11, p. 9-29.

2007 Les pompes de cale et l'évacuation de l'eau de sentine sur les navires antiques, dans J.-P. Brun et J.-L. Fiches (éds), Énergie hydraulique et machines élévatrices d'eau dans l'Antiquité (Actes du Colloque International, Vers - Pont du Gard, 20-22 septembre 2006), Naples, Centre Jean-Bérard, p. $51-66$.

CArre M.-B., JÉZÉGou M.-P.

1984 Pompes à chapelet sur des navires de la fin de l'Antiquité et du début du Moyen Âge, Archaeonautica, 4, p. 115-143.

18. Dans l'article, l'auteur l'identifie comme une tête de maillet, ce qui paraît tout à fait improbable à cause de l'engoujure de la base et de l'absence de mortaise permettant la fixation du manche.
CArre M.-B., Rival M.

1988 Le gréement et l'équipement, dans A. Hesnard et al., L'épave romaine Grand Ribaud D (Hyères, Var), éditions du CNRS, Paris (Archaeonautica, 8), p. 113-117.

CASSON L.

1971 Ship and Seamanship in the Ancient World, Baltimore/London John Hopkins, University Press (réédition 1995).

Charlin G., Gassend J. M., Lequément R.

1978 L'épave antique de Cavalière (Le Lavandou, Var), Archaeonautica, 2, p. 9-93.

Colls D., Etienne R., Lequément R., Liou B., Mayet F.

1977 L'épave Port-Vendres II et le commerce de la Bétique à l'époque de Claude, Paris, éd. du CNRS (Archaeonautica, 1).

Cornelio Cassai C.

1990 Attrezzature e armamento, dans F. Berti (éd.), Fortuna Maris, La nave romana di Comacchio, Bologne, Nuova Alfa editoriale, p. 43-52.

DeVILlers G.

1971 Manuel de matelotage et de voilure, Paris, ed. Martimes et d'Outremer.

HESNARD A.

2012 L'épave La Madrague de Giens (Var) et la plaine de Fondi (Latium). Producteurs des vins, des amphores Dr. 1b et commerçants, Archaeonautica, 17, p. 71-93.

JONCHERAY J.-P

1975 L'Épave « $C$ » de la Chrétienne (Cahiers d'archéologie subaquatique, supplément 1). 
Oleson J. P.

1983 A Roman sheave block from the harbour of Caesarea Maritima, Israel, IJNA, 12.2, p. 155-170.

\section{PIERCY R.C.M.}

1977 Monbasa wreck excavation, preliminary report 1977, IJNA, 6.4, p. 113-124.

PoMey P.

1982 Le navire romain de la Madrague de Giens, CRAI, JanvierMars, p. 113-154.

PoMeY P. (DIR.)

1997 La navigation dans l'Antiquité, Aix-en-Provence, Edisud.

Swiny, H. W., KatZev, M. L.

1973 The Kyrenia Shipwreck: a fourth-century B.C. Greek merchant ship, dans D. J. Blackman (ed.), Marine Archaeology, Londres, Butterworth (Colston Papers, 23), p. 339-355.
TChernia A.

1988 Les Urinatores sur l'épave de La Madrague de Giens, Cahiers d'histoire, XXXIII, 3-4, p. 489-499.

Tchernia A., Pomey P., Hesnard A.

1978 L'épave romaine de la Madrague de Giens (Var), Paris, Éditions du CNRS (Gallia, suppl. 34).

UCELLI G.

1950 Le navi di Nemi, Rome, Istituto Poligrafico e Zecca dello Stato (1éd. 1940).

WhiteWRight J.

2007 Roman Rigging Material from the Red Sea Port of Myos Hormos, IJNA, 36.2, p. 282-292.

Ximénès S., Moerman M.

1990 Port romain des Laurons II : éléments d'accastillage antiques, Cahiers d'Archéologie Subaquatique, 9, p. 5-25. 